\title{
COMPARISON OF THEORY AND IN SITU OBSERVATIONS FOR ELECTRON AND ION DISTRIBUTIONS IN THE NEAR WAKE OF THE EXPLORER 31 AND AE-C SATELLITES
}

\author{
URI SAMIR $\dagger$ and ERNEST G. FONTHEIM* \\ *Space Physics Research Laboratory, Department of Atmospheric and Oceanic Science, The University of Michigan, \\ Ann Arbor, MI 48109, U.S.A.
}

†Department of Geophysics and Planetary Sciences, Tel-Aviv University, Ramat-Aviv, Israel

(Received 24 February 1981)

\begin{abstract}
Measurements of electron density, plasma potential, and mean ion mass from the Explorer 31 satellite and measurements of ion current, plasma potential, and ion composition from the Atmosphere Explorer $\mathrm{C}$ (AE-C) satellite were used in a comparative study with theory regarding the charged particle distribution in the near wake of an ionospheric satellite. The theoretical wake model of Parker (1976) has been used in the study. It is shown that theory and experiment agree fairly well in the angle-of-attack range between 90 and $135^{\circ}$. In that angular range even the neutral approximation (which treats ions as if they were neutral particles thus ignoring the influence of the electric field) gives fair agreement with the measurements. In the maximum rarefaction zone $\left(145<\theta<180^{\circ}\right.$ ), however, the theoretical model overestimates the measured ion depletion (AE-C measurements) by several orders of magnitude. A similar conclusion is drawn from the comparison between theory and the Explorer 31 electron measurements where the theory also significantly overestimates the electron depletion. The study indicates that the discrepancies are mainly due to the use of a steady-state theory and of a single ion equation (using a mean ion mass). It is recommended that improved agreement between theory and experiment be obtained by the use of the timedependent Vlasov-Poisson equations with separate equations for the various ion species.
\end{abstract}

\section{INTRODUCTION}

It is known that the motion of a satellite through the terrestrial rarefield plasma creates severe disturbances in the satellite vicinity. The disturbed zones may reach large distances downstream and elsewhere around the satellite. Behind the satellite a region of rarefaction is created in which electrons and ions are unequally depleted. The amount of depletion in this region as well as other characteristic features (e.g. current enhancements, trailing shocks, plasma oscillations and instabilities, see Gurevich et al., 1973; Gurevich and Pitaevski, 1975; Al'pert, 1976) depend strongly on body and plasma parameters among which we cite the parameters:

$$
\begin{gathered}
R_{D}=\frac{R_{0}}{\lambda_{D}}, \quad T=\frac{T_{e}}{T_{+}} \\
S=\frac{V_{S}}{\left(2 K T_{e} / M_{+}\right)^{1 / 2}}, \quad \phi_{N}=\frac{e \phi_{s}}{K T_{e}},
\end{gathered}
$$

where $R_{0}$ is the effective radius of the satellite, $\lambda_{D}$ is the Debye length, $T_{e}$ and $T_{+}$are the electron and ion temperatures, respectively, $V_{S}$ is the satellite velocity, $M_{+}$is the average ion mass, $\phi_{s}$ is the satellite potential with respect to the plasma, $e$ is the electron charge, and $K$ is Boltzmann's constant. The interaction between the satellite and the terrestrial plasma is of interest in its own right and also since it is possible to see the satellite as a "model" for the more general rarefield plasma flow interaction with non-magnetized bodies in the solar system. In fact, the investigation of the flow interactions of the terrestrial ionosphere with orbiting satellites is an example for the plasmabody interaction in a supersonic and sub-Alf venic flow regime. Similar flow regimes are known to exist in the outer solar system where Galilean satellites orbit around their parent planets, e.g. Io around Jupiter and Titan around Saturn, etc.

Although the investigation of various aspects of satellite-ionosphere interactions is not new, the numerous theoretical studies available at present (e.g. Gurevich et al., 1969; Liu, 1969, 1975; Gurevich and Pitaevski, 1975; Grabowsky and Fisher, 1975; Call, 1969; Fournier, 1971; Parker, 1976; Al'pert, 1976) do not yield a generally accepted theory which can adequately describe the distribution of charged particles and local potential around an ionospheric satellite in particular in the region of maximum rarefaction on the wake axis. This is due mainly to the well-known difficulties of achieving a self-consistent solution of the Vlasov- 
Poisson equations for practical boundary conditions and plasma parameters. The available theories differ mathematically in the numerical approaches adopted in order to obtain approximate solutions. More important, the theories of ten employ physical assumplions whose validity and range of applicability to satellite-ionosphere interaction are subject to controversy. A possible way of gaining a good physical insight into the degree and range of validity and applicability of the assumptions used in the theoretical models is via theory-experiment comparisons. Such comparisons, restricted and imperfect as they may be, should be performed using in situ measurements conducted for the widest possible range of plasma parameters. Unfortunately, no such studies have been performed in an extensive manner, except for the studies of Gurevich et al. (1969), Samir and Jew (1972), Gurevich and Dimant (1975), and Samir et al. (1975)., all of which used a relatively small sample of in situ measurements under a restricted range of plasma parameters. A major reason for this shortcoming is the limited amount of in situ measurements available at the present time for such studies. The latter situation stems from the fact that most of the published data relating to satellite-ionosphere interactions are byproducts of experiments designed to measure the ambient ionosphere by avoiding "wake-affected" data. It is surprising that the study of wakes behind bodies has not yet widely been recognized as part of the basic physical problem of an expansion of a plasma into a vacuum which is of fundamental interest to space plasma physics.

In the present investigation the Parker wake model is being compared with in situ measurements of electron and ion distributions in the wakes of the Explorer 31 and Atmosphere Explorer C(AE-C) satellites.

The objective of this study is three-fold: (i) to provide a comparison between the results of the Parker model (Parker 1976, 1977) and measurements carried out around a satellite in a planetary ionosphere which may help in establishing criteria for the applicability of the Parker model to real cases of satellite-plasma interactions; (ii) to obtain an improvement in our understanding of the reliability and quality of low energy particle measurements; and (iii) to provide information of use for the planning of instrument location on ejectable ensembles of probes (e.g. subsatellites of various kinds) and on the Spacelab itself in future shuttle Spacelab missions. Such information should be useful for experiments in the area of solar system plasma physics, particularly dealing with flow interactions in supersonic, sub-Alfvénic flow regimes.

\section{BRIEF REVIEW OF THE PARKER WAKE MODEL}

Since the physical assumptions underlying Parker's model and the computational scheme have been discussed in great detail elsewhere (Parker, 1976, 1977), only those aspects of the model will be briefly summarized here which are deemed necessary for an understanding of this paper. The model is based on the following physical assumptions:

(1) Flow is collisionless.

(2) Effect of geomagnetic field on particle motion is negligible.

(3) Both electrons and ions have non-vanishing temperatures, and their thermal motion is included.

(4) The electron density distribution is given by the Boltzmann factor $\exp \left(\phi_{N}\right)$.

(5) Charged particles incident on the body surface are neutralized.

(6) No emission of particles from the surface (photuelectrons, secondary electrons).

(7) The body is a perfect conductor.

(8) Steady state.

The Parker computer program solves the simultaneous Vlasov and Poisson equations under the above named assumptions by tracing particles trajectories backward from points near the body to infinity. The boundary condition at "infinity" is represented by a so-called floating condition, i.e. a linear relation between the potential $\phi$ and its gradient, where the point at "infinity" $Z_{\max }$, is chosen sufficiently far so that the calculated quantities are insensitive to changes in $Z_{\max }$. In the present study this relation is taken to be the same as that for a Coulomb potential. According to Parker the exact relation is not important when the external boundary of the grid is sufficiently far away.

In the zero-th iteration a certain ion density distribution is assumed. In this study the neutral approximation has been used as a starting point for the ions, i.e. the ions are treated as neutral particles thus ignoring electric field effects, while the electrons are assumed to have a Boltzmann distribution. The Poisson equation is solved with the zero-th iteration charge densities yielding the first iteration potential distribution which is then substituted into the Vlasov equation from which the first iteration charged particle densities are obtained. This iterative procedure is repeated until any desired convergence has been achieved. 
3. DISCUSSION OF EXPERIMENTS USED IN THE COMPARISON

For the purpose of conducting a comparative study of experiment with theory we have used measurements of electron density, electron temperature, plasma potential and average ion composition from the Explorer 31 satellite (Samir and Jew, 1972) and measurements of ion current, electron temperature plasma potential and ion composition from the AE-C satellite (Samir et al., 1979).

The Explorer 31 measurements used in the present investigations were made by a planar, guarded electron probe which was flush-mounted on the surface of the satellite (see Fig. 1a). The experimental technique is that used by the Mullard Space Science Laboratory, University College London. Details on the method of data analysis and the use of these measurements in theoryexperiment comparisons are given by Samir and Jew (1972), Willmore (1970) and Wrenn (1969).

The AE-C measurements used in the present investigation were made by the cylindrical electrostatic probe (CEP) and the Bennett ion mass spectrometer (BIMS). Details on the probe and spectrometer techniques and methods are given by Brace et al. (1973) and Brinton et al. (1973) respectively. The CEP probe (see Fig. 1b) provided measurements of ion and electron currents, electron temperature and satellite potential (with res-

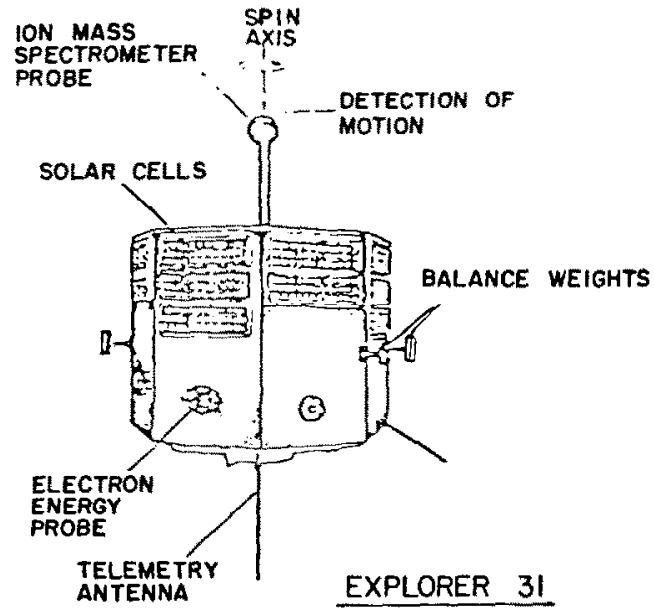

(a)

PROBE ON THE SATELLITE:

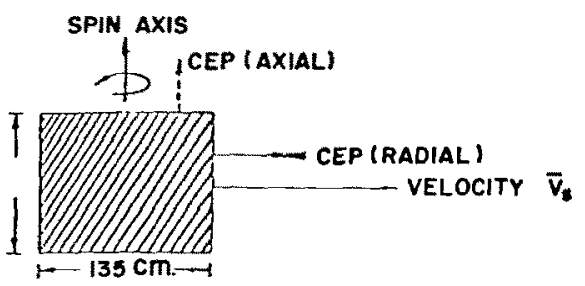

(b)

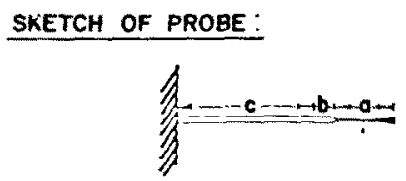

a collector $* 7.5 \mathrm{~cm}$

v GUARD $=2.5 \mathrm{~cm}$

c short BOOM $25 \mathrm{~cm}$

DIAMETER OF COLLECTOR $* 0.2 \mathrm{~cm}$

ATMOSPHERE EXPLORER C

Fig. 1. Probe locations on board the (a) Explorer 31 satellite and (b) AE-C satellite. 


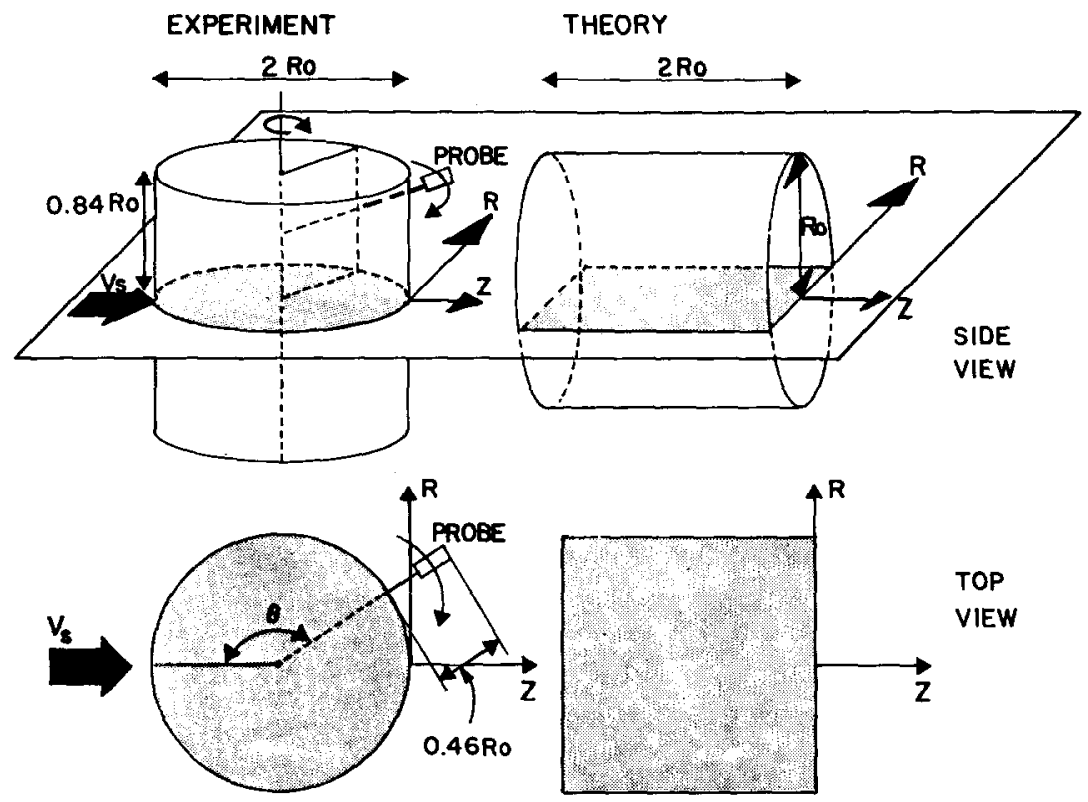

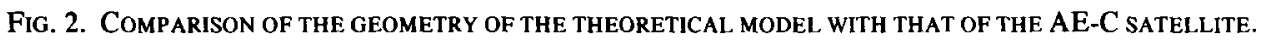

pect to the plasma) at a distance of about $0.5 \boldsymbol{R}_{0}$ from the surface of the satellite (where $R_{0}=$ satellite radius), The measurements of ion composition were made by the BIMS experiment. Further details on the use of those AE-C measurements for wake studies are given in Samir et al. (1979).

It is important to emphasize that the Explorer 31 data provide electron densities, while the AE-C data yield ion and electron currents.

The geometry of the theoretical model is compared to that of the AE-C satellite in Fig. 2. The situation for the Explorer 31 satellite is very similar except that the probe is flush-mounted on its surface. As the figure indicates, the axis of the theoretical model is parallel to the flow vector while the satellite axis is perpendicular to it. Hence, the projection of the theoretical model on the plane perpendicular to the flow vector $\mathbf{v}_{s}$ presents an area of magnitude $\pi R_{0}^{2}$ and that of the satellite an area of $3.36 R_{0}{ }^{2}$. The depth (in the direction of the flow) is $2 R_{0}$ for both the satellite and the model. Since the depths are the same in both cases, and since the cross-section which the satellite and the model present to the flow agree within $10 \%$, the dimensions of the theoretical model are sufficiently close to those of the satellite for a meaningful comparison between measurements and theory to be carried out-especially in view of uncertainties in the calculations and the estimated measurement errors.

\section{RESULTS AND DISCUSSION}

Figure 3(a) shows the locations of the ion current observation points and of the numerical grid points at which the densities have been calculated for comparison with the AE-C data. The two sets of points are generally close enough to each other to permit meaningful comparisons of measured and calculated quantities within the accuracy of the measurements although the third and fourth computational grid points from the left are considerably closer to the body than the corresponding observation points. For this reason some disagreement between calculated quantities at those points and the measured quantities at the corresponding observation points could be expected. Figure 3(b) shows the locations of the electron density observation and numerical grid points for comparison with the Explorer 31 data. In this case the two measurement points at the largest angles of attack are much less shielded from the flow than the corresponding grid points of the calculation. This is expected to affect the calculated results as will be shown below.

In Table 1 the relevant experimental data and plasma flow parameters are listed. Here $\theta$ is the angle of attack defined in Fig. 2. The numerical values are the averages over the various altitude ranges given in the second column. The data listed in the last column refer to the maximum angle of attack for which measurements are available. Ac- 


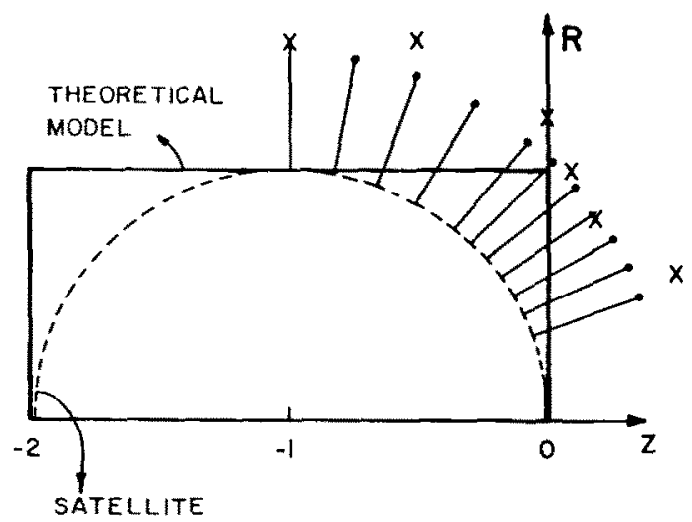

(a)

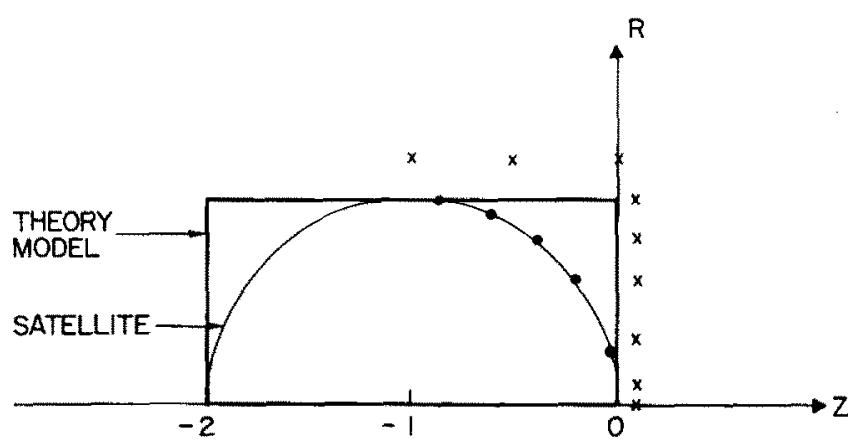

(b)

Fig. 3. Locations OF NUMERICAL ORID POINTS OF CALCULATION $(x)$ AND OF MEASUREMENT POINTS $(\cdot)$ FOR THE CASE OF THE (a) AE-C SATELLITE AND (b) EXPLORER 31 SATELLITE.

cording to Table 1 cases 1, 3, and 4 of the Explorer 31 data have approx. equal average ionic Mach numbers $\left(S_{A V}\right)$, which is about half the value of cases $1,2,3$, and 6 of the AE-C data. On the other hand, the average value of the normalized potential $\phi_{N}\left(\equiv e \phi_{s} / k T_{e}\right)$ for the three Explorer 31 cases is about half of the average potential of the AE-C cases. The data from the two satellites also differ in the ion composition. The mean ion mass in cases 1, 3, and 4 of the Explorer 31 data is $\sim 9.2$ while that in cases $1,2,3$, and 6 of the AE-C data is approx. 15.8. Another significant difference between the Explorer 31 and $A E-C$ parameters lies in the body size $R_{D}$. While $R_{D}<10$ for all of the Explorer 31 cases, $R_{D}>50$ for all of the AE-C cases and even $R_{n}>100$ for two of them. Thus the Explorer 31 data represent an interaction between a plasma and a relatively small body while the $\mathrm{AE}-\mathrm{C}$ data represent an interaction between a plasma and a large body. We will consider here $R_{D}<10$ to represent the "small body" case and
$R_{D}>50$ to represent the "large body" case. Both cases are of interest in connection with the theory of probe and body interactions with planetary ionospheres.

In Fig. 4 the normalized current profiles of the above mentioned four AE-C cases are compared with the calculated normalized density profile obtained with the neutral approximation. Since $\lambda_{D}$ is infinite (i.e. $R_{D}=0$ ) in the neutral approximation, the same computed profile describes all four cases. As seen there is a significant difference between the measured current ratios and the computed "neutral" density ratios. In fact the difference starts at $\theta \sim 125^{\circ}$ and becomes most significant for $\theta>147^{\circ}$. A similar behaviour is shown in Gurevich et al. (1969) and Alpert (1976) using analytical expressions for the neutral approximation. The differences between cases 1,2,3 and 6 of the AE-C measurements are due to the differences in $\boldsymbol{R}_{D}$ (see also Table 1) as discussed in Samir et al. $(1979,1980)$. It is, however, interesting 
TABLE 1. EXPERIMENTAL DATA AND SOME PLASMA PARAMETERS*

EXPLORER 31

\begin{tabular}{ccccccccccc}
\hline & \multicolumn{1}{c}{} & & & & & \multicolumn{2}{c}{$\begin{array}{c}\text { Measurement at } \\
\text { maximum } \\
\text { wake position }\end{array}$} \\
\hline $\begin{array}{c}\text { Case } \\
\text { No. }\end{array}$ & $\begin{array}{c}\text { Altitude range } \\
(\mathrm{km})\end{array}$ & $\begin{array}{c}T_{e} \\
(\mathrm{~K})\end{array}$ & $\begin{array}{c}N_{e} \\
\left(\mathrm{~cm}^{-3}\right)\end{array}$ & $\begin{array}{c}\phi_{s} \\
(\mathrm{~V})\end{array}$ & {$\left[M_{i}\right]_{A V}$} & $R_{D}$ & $S_{A V}$ & $e \phi_{s} / K T_{e}\left[\frac{N_{c}\left(\theta=165^{\circ}\right)}{N_{e}\left(\theta=90^{\circ}\right)}\right]$ \\
\hline 1 & $709-1109$ & 2667 & $3.79 \times 10^{3}$ & -0.998 & 8.5 & 6.9 & 3.4 & -4.3 & $(2.2 \pm 1.8) \times 10^{-1}$ \\
2 & $1242-1693$ & 2968 & $1.48 \times 10^{3}$ & -1.007 & 1.2 & 4.1 & 1.1 & -3.9 & $(5.1 \pm 2.0) \times 10^{-1}$ \\
3 & $696-1071$ & 2292 & $4.15 \times 10^{3}$ & -1.097 & 8.0 & 7.8 & 3.6 & -5.5 & $(1.2 \pm 0.5) \times 10^{-1}$ \\
4 & $909-605$ & 3038 & $8.24 \times 10^{3}$ & -1.004 & 11.0 & 9.5 & 3.7 & -3.8 & $(1.0 \pm 0.3) \times 10^{-1}$ \\
\hline
\end{tabular}

AE-C

Measurement at maximum Plasma properties Plasma parameters wake position

\begin{tabular}{cccccccccc}
\hline $\begin{array}{c}\text { Case } \\
\text { No. }\end{array}$ & $\begin{array}{c}\text { Altitude range } \\
(\mathbf{k m})\end{array}$ & $\begin{array}{c}T_{e} \\
(\mathrm{~K})\end{array}$ & $\begin{array}{c}N_{e} \\
\left(\mathrm{~cm}^{-3}\right)\end{array}$ & $\begin{array}{c}\phi_{s} \\
(\mathrm{~V})\end{array}$ & {$\left[M_{i}\right]_{A V}$} & $R_{D}$ & $S_{A V}$ & $e \phi_{s} / K T_{e}$ & {$\left[\frac{I_{+}\left(\theta=160^{\circ}\right)}{I_{+}\left(\theta=90^{\circ}\right)}\right]$} \\
\hline 1 & $276-313$ & 970 & $2.65 \times 10^{5}$ & -0.802 & 16.0 & 135.7 & 8.0 & -9.4 & $(7.3 \pm 0.5) \times 10^{-3}$ \\
2 & $360-404$ & 977 & $1.93 \times 10^{5}$ & -0.816 & 16.0 & 116.3 & 8.0 & -9.5 & $(1.4 \pm 0.1) \times 10^{-2}$ \\
3 & $450-508$ & 948 & $9.87 \times 10^{4}$ & -0.770 & 16.0 & 83.8 & 8.0 & -9.3 & $(2.1 \pm 0.1) \times 10^{-2}$ \\
4 & $558-600$ & 991 & $4.89 \times 10^{4}$ & -0.755 & 9.6 & 58.0 & 5.9 & -8.7 & $(1.0 \pm 0.5) \times 10^{-1}$ \\
5 & $357-411$ & 1039 & $4.02 \times 10^{5}$ & -0.771 & 16.0 & 162.5 & 7.7 & -8.4 & $(7.8 \pm 1.9) \times 10^{-3}$ \\
6 & $475-538$ & 948 & $7.48 \times 10^{4}$ & -0.732 & 15.1 & 73.4 & 7.8 & -8.8 & $(0.7 \pm 0.3) \times 10^{-1}$ \\
\hline
\end{tabular}

*After Samir and Jew (1972) and Samir et al. (1979).

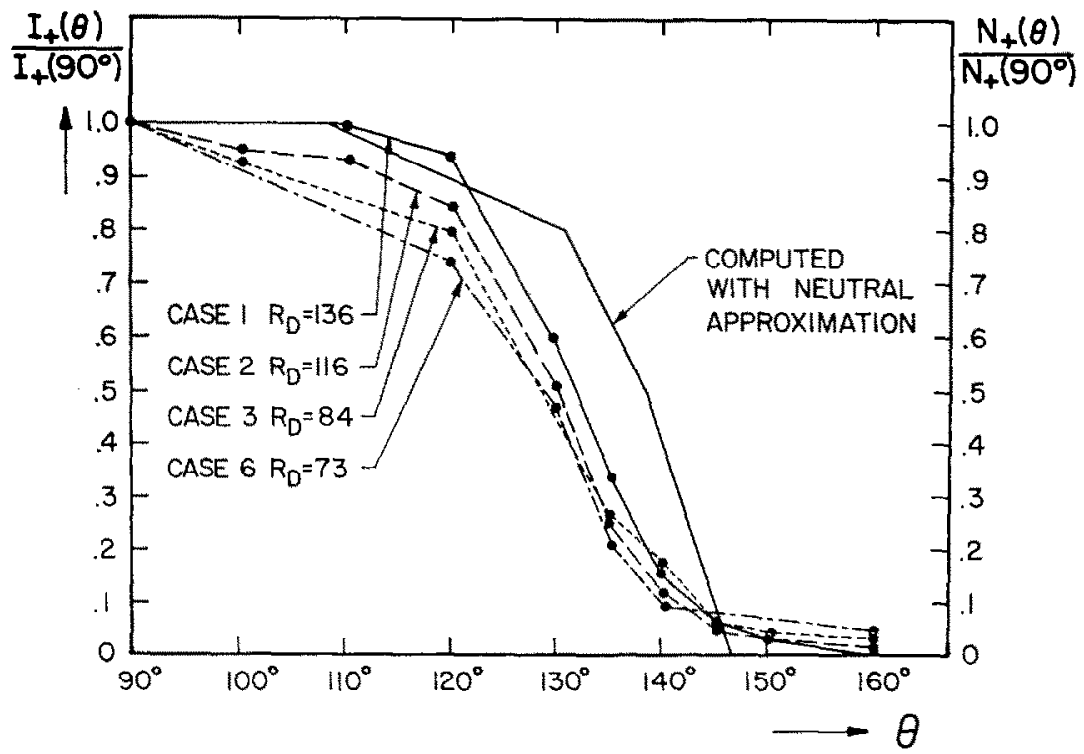

Fig. 4. COMparison OF AE-C MEASURED ION CURRENT PROFLles With the NEUTRAL APPROXimation. The selected profiles have approximately equal Mach numbers and normalized satellite potentials and differ in normalized body radii (see Table 1). The neutral approximation curve goes along the axis for $\theta>146^{\circ}$. 
to note that case 1 , i.e. the case of largest $R_{D}$, is the closest to the neutral approximation.

It should be borne in mind that while the measurements give current values, the calculation yields densities. Both have been normalized to their respective values at $90^{\circ}$ for the purpose of this comparative study. The relation between the ion density and ion current ratios in the wake has recently been investigated by Samir et al. (1980) who showed that those ratios are approximately equal for the range $90 \leq \theta \leq\left(150-160^{\circ}\right)$. This is also in general agreement with the conclusion drawn earlier by Gurevich et al. (1969). Hence, while ideally it would be preferable to compare calculated ion density ratios with measured ones or, alternatively, calculated ion current ratios with measured ones, we can only work with the data available to us.

The curves show that for $\theta \leq 138^{\circ}$ the normalized ion current slowly increases with increasing $R_{D}$, and the measured values lie below the neutral approximation curve (except for two points of case 1). Hence, the neutral approximation underestimates the true ion depletion in this angular region. For $\theta \geq 146^{\circ}$, the normalized ion current decreases with increasing $R_{D}$, and the measured values lie above the neutral approximation curve. Therefore in this region the neutral approximation overestimates the measured ion depletion. The region between 137.7 and $146.3^{\circ}$ is a transition region. The reason why the neutral approximation underestimates the ion depletion in the region $\theta \leq 138^{\circ}$ and overestimates it for $\theta>146^{\circ}$ (both at a distance $0.5 R_{D}$ from the surface of the body) can be explained by the behaviour of the potential distribution. The neutral approximation is equivalent to $\phi=0$ everywhere.
When the calculation of the potential due to charge separation and its effect on the electron and ion distributions (as outlined in Section 2) is included, then it turns out that for angles in the range $\theta \leq 125^{\circ}$ the potential is positive with respect to the undisturbed plasma. This has the effect of "bending" the ion trajectories away from this region thus resulting in a larger ion depletion than predicted by the neutral approximation. On the other hand, in the region $\theta \geqslant 125^{\circ}$ and particularly for $\theta \geqslant\left(140-150^{\circ}\right)$ the potential turns out to he negative, thus attracting additional ions into a region which is almost inaccessible to the straightline neutral trajectories because of gcometrical shielding by the body. Therefore, here the neutral approximation overestimates the ion depletion.

The behaviour of the normalized ion current as a function of $R_{D}$ at a large angle of attack $\left(\theta=160^{\circ}\right)$ is shown in Fig. 5. This figure demonstrates more explicitly than Fig. 4 that at large angles the normalized ion current decreases with increasing $R_{D}$ (all other parameters being kept approximately constant). This conclusion has been further substantiated in Samir et al. (1980) for a wider range of $R_{D}$ (i.e. $\left.37 \leq R_{D} \leq 247\right)$. Cases $4\left(R_{D}=58\right)$ and 5 $\left(R_{D}=162\right)$ have been included in this figure although they deviate (particularly case 4) more from the averages of $S$ and $\phi_{N}$ than do the other cases. However, their values of $S$ and $\phi_{N}$ are sufficiently close to the other ones so as to generally follow the same exponential curve as the rest of the points (see also Samir et al., 1979, 1980).

The comparison between the measured profiles and the neutral approximation can be summarized by stating that for all values of $\theta$ the plasma flow close to the body approaches the

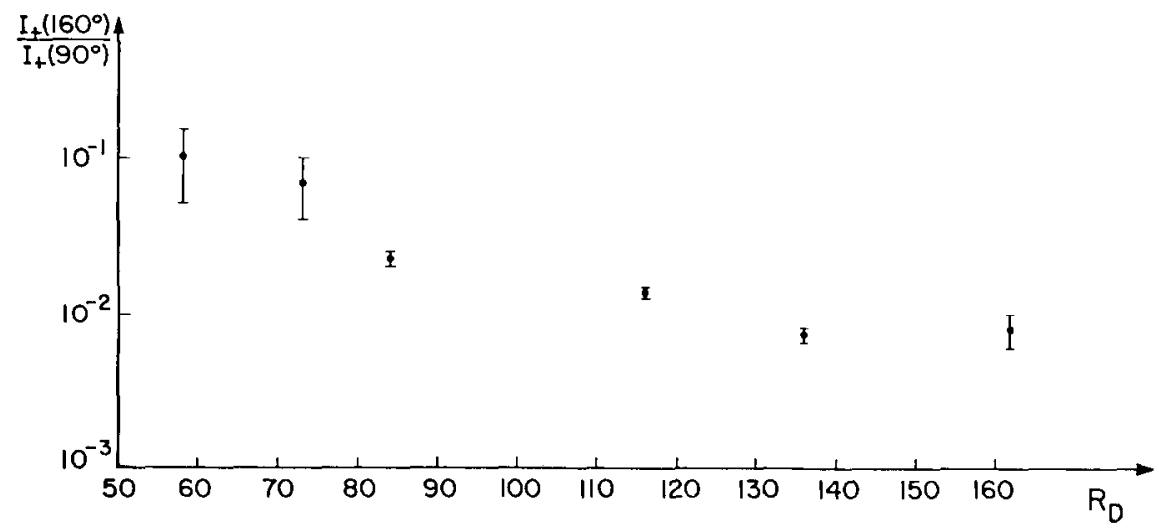

FIG. 5. VARIATION OF THE AE-COBSERVED NORMALIZED CURRENT RATIO AT THE ANGLE OF ATTACK OF $160^{\circ}$ AS A FUNCTION OF NORMALIZED BODY RADIUS. 
neutral approximation with increasing $R_{D}$. This makes physical sense, for the larger the body radius $R_{0}$ is compared to the Debye length $\lambda_{D}$ the less the plasma flow close to the body is expected to be governed by charge separation effects. Such effects will be most important if $R_{0}$ is of the order of $\lambda_{D}$.

An interesting comparison is presented in Figs. 6 and 7 . In these figures the measured angular profiles are shown together with the 0th (neutral approximation) and 15 th iterations for cases 5 (Fig.
6) and 6 (Fig. 7) with $R_{D}=162$ and $R_{D}=73$ respectively. AE-C cases 1,2 and 3 fall in between these two values of $R_{D}$ while showing basically equal values of $S_{A V}$ and $\phi_{N}$. We find that for both of the above cases, the iteration 15 profiles are closer to the experimental profile (in the angular range $90 \leq$ $\theta \leq 147^{\circ}$ ) than is the neutral approximation profile (i.e. iteration 0 th). The largest relative discrepancies between measured and calculated values occur in the maximum rarefaction region $\left(\theta>147^{\circ}\right)$. This is shown in Table 2. The neutral

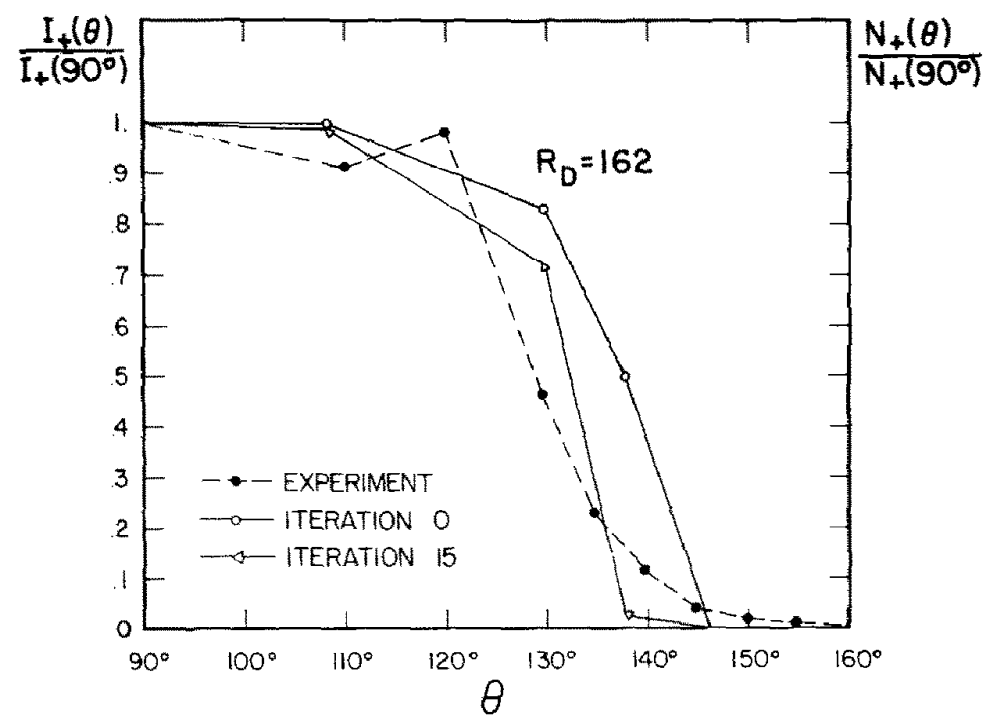

Fig. 6. COMPARISON OF OTH ITERATION (NEUTRAL APPROXIMATION), 15TH ITERATION, AND MEASURED PROFILE FOR AE-C CASE 5.

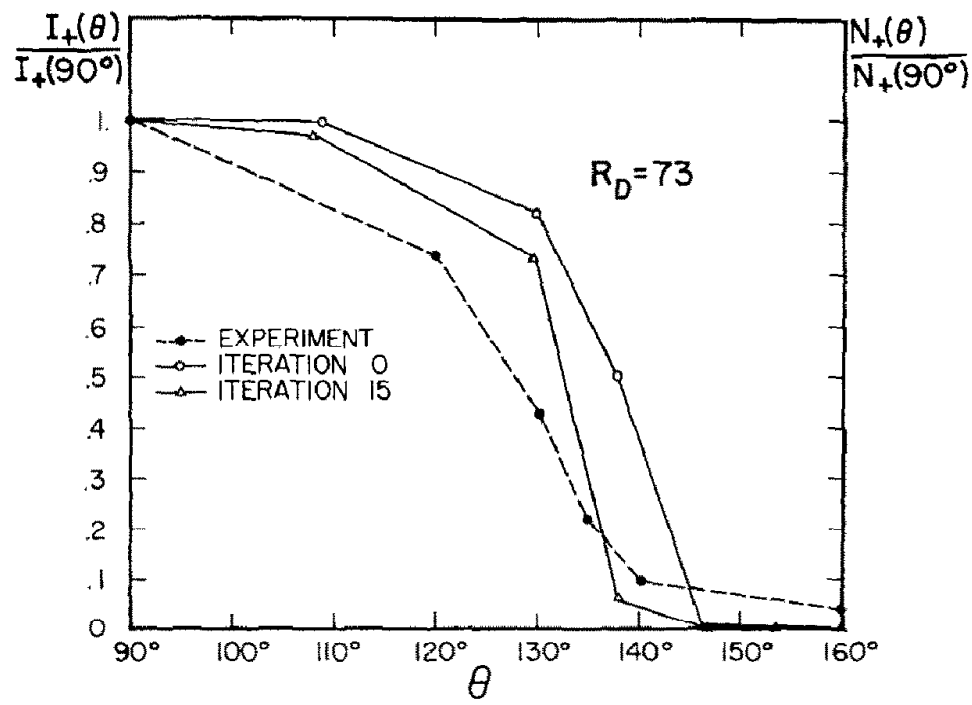

FIG. 7. COMPARISON OF OTH TTERATION (NEUTRAI APPROXIMATION). 15TH ITERATION, AND MEASURED PROFILE FOR AE-C CASE 6. 


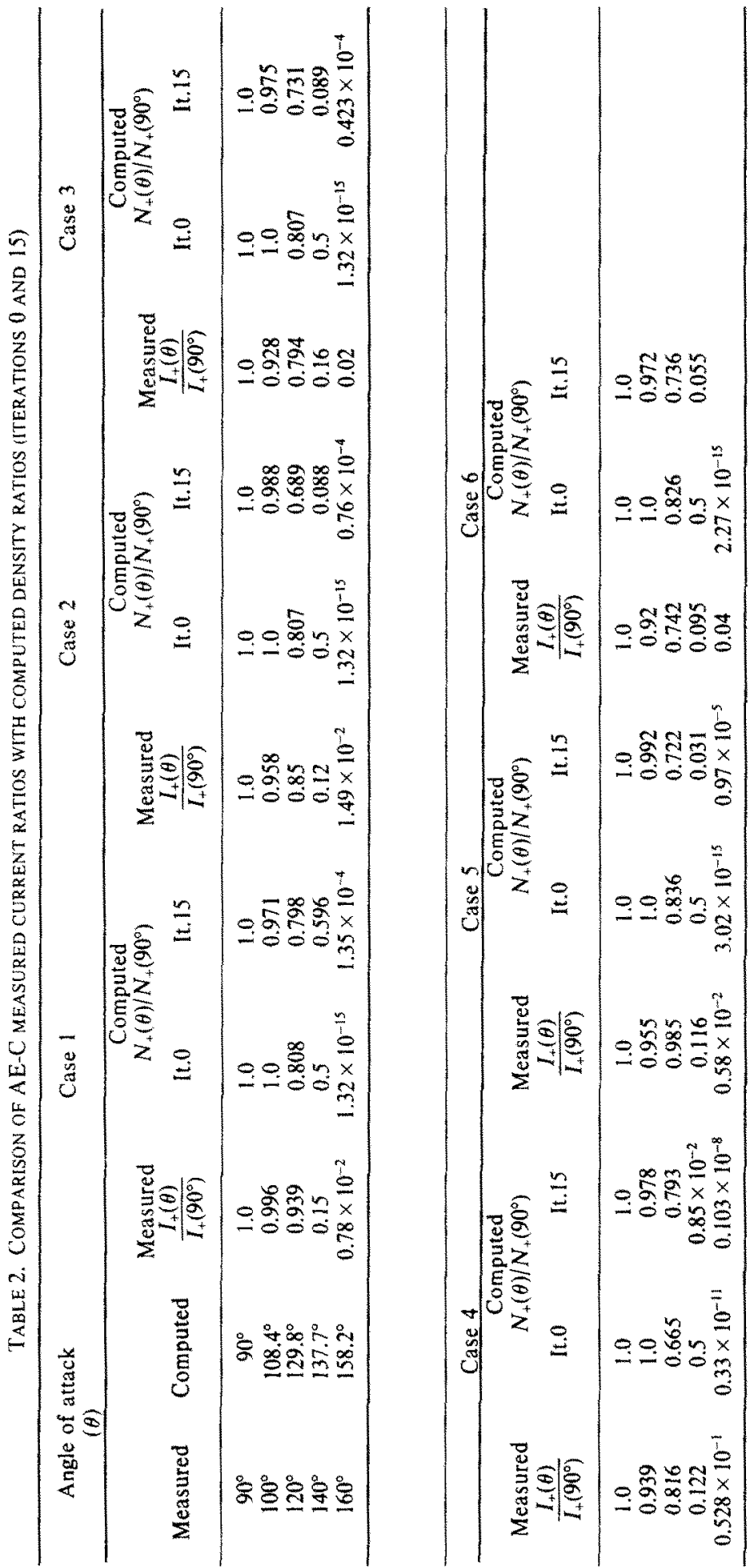


approximation differs by more than 10 orders of magnitude from the measured values, and even the 15 th iteration values are by 2 to 3 orders of magnitude smaller than the observed values. The results of these calculations are therefore not significantly better than those of Gurevich et al. (1969) and Al'pert (1976) who used a somewhat different version of the neutral approximation but with a considerably simpler mathematical formulation. The discrepancy between theory and experiment in the maximum rarefaction region, which has been known for sometime, has therefore not been resolved by the much more elaborate theory of Parker.

The fact that Parker's model does not substantially improve the disagreement between theory and experiment in the deep wake may be an indication that steady-state models cannot be further pushed and that time-dependent solutions which consider the existence of accelerating mechanisms in the wake (e.g. Gurevich et al., 1973; Gurevich and Pitaevski, 1975) as well as the existence of plasma oscillations and wave-particle interactions (Al'pert, 1976) should be sought. We propose that future experimental and theoretical studies in this area should proceed along these lines.

In Fig. 8 the measured and calculated normalized electron density profiles for the four Explorer 31 cases are compared. The 0th iteration is of no interest for electrons since this is merely the Boltzmann distribution for $\phi=0$ as mentioned in Section 2.

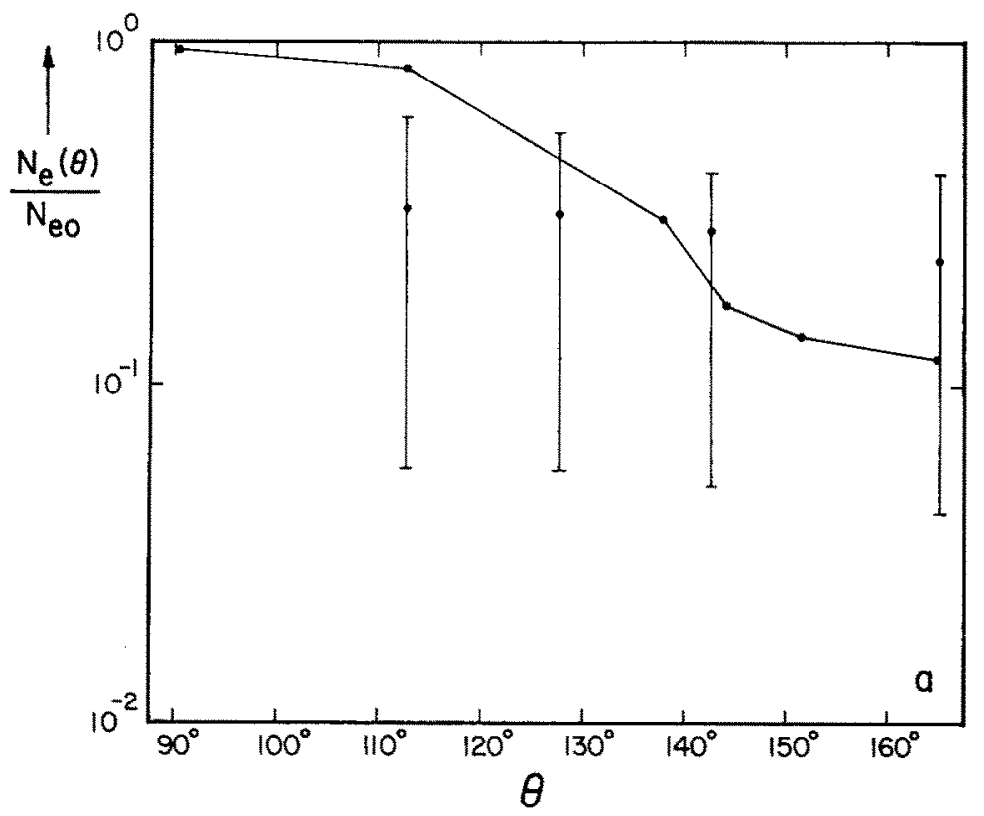

(a)

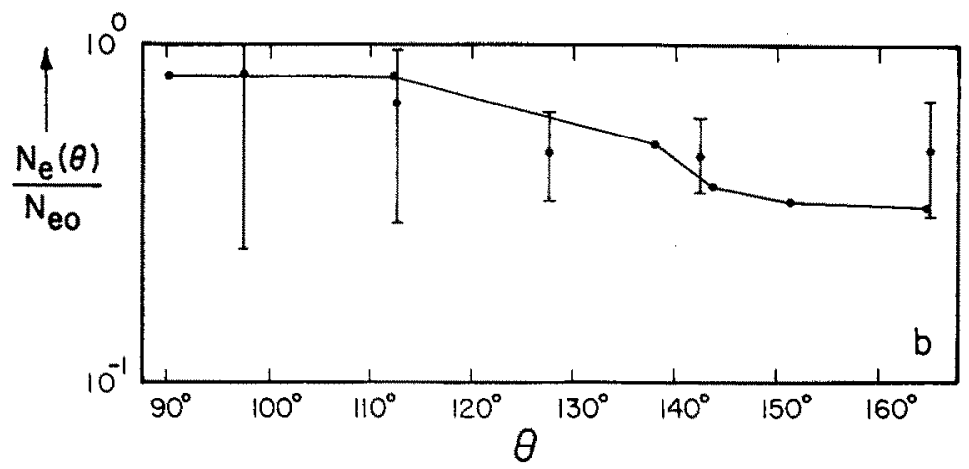

(b) 


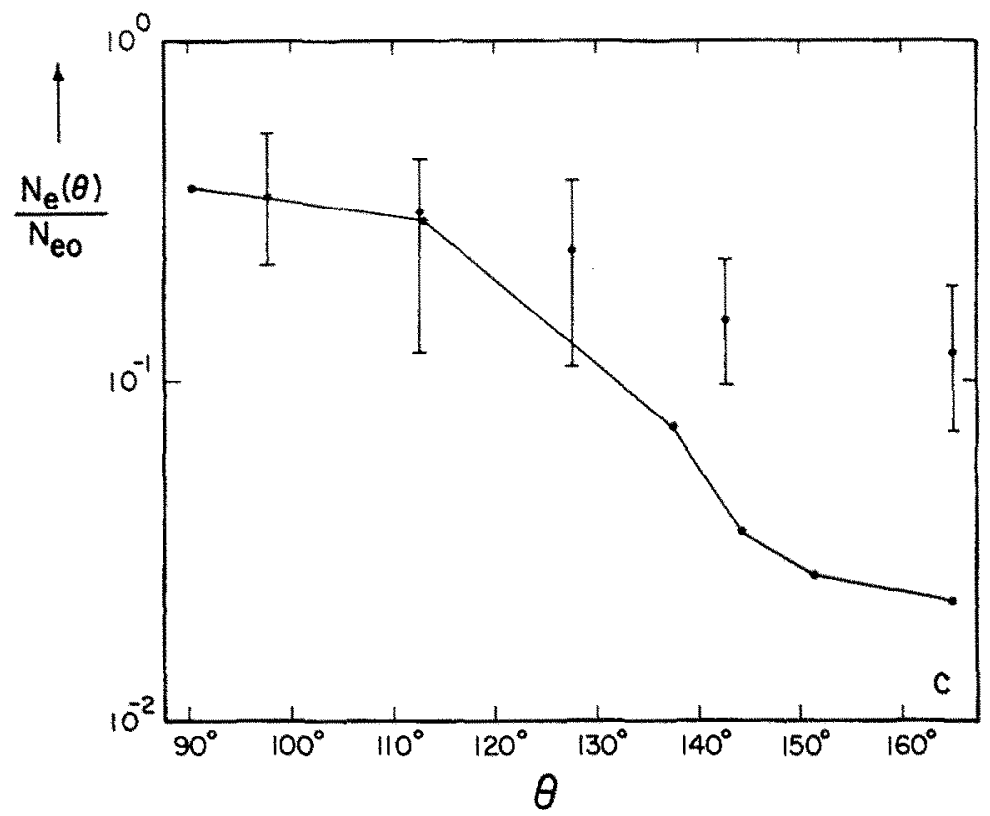

(c)

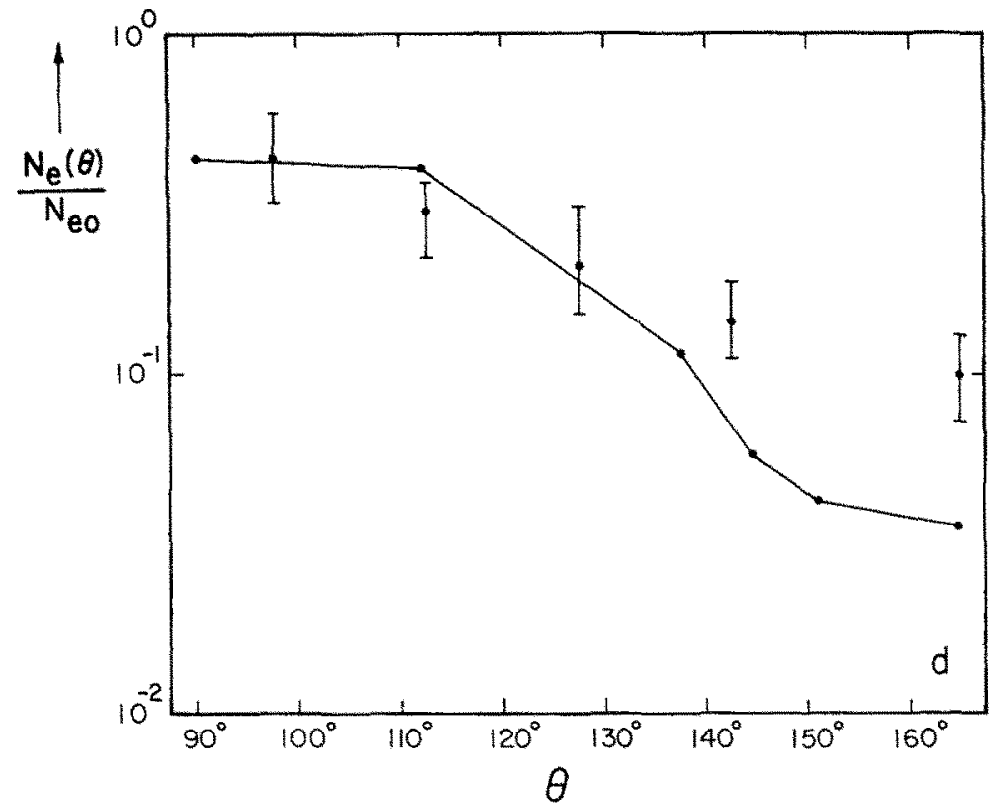

(d)

Fig. 8. Comparison of Calculated (POINTS along CURVE) and OBSERved (POINTS WITH erRor bars) ELECTRON DENSITY RATIOS FOR EXPLORER 31 (a) CASE 1, (b) CASE 2, (c) CASE 3, (d) CASE 4.

As seen from Figs. 8(a) and (b) the degree of agreement between theory and experiment for cases 1 and 2 is moderate. For cases 3 and 4 , however, there seems to be a consistent difference such that the calculated values of $\left|\left(N_{e}(\theta)\right) /\left(N_{e}\right)\right|$ are lower than the measured ones particularly for $\theta>130^{\circ}$. This behaviour is basically similar to what was obtained for $\left|\left(I_{+}(\theta)\right) /\left(I_{+}\left(90^{\circ}\right)\right)\right|$ vs cal- 
culated $\left|\left(N_{+}(\theta)\right) /\left(N_{+}\left(90^{\circ}\right)\right)\right|$ for the AE-C data (see Fig. 4 and Table 2). There are several reasons for the above differences between theory and observation, particularly in the maximum rarefaction region (i.e. $\theta>150^{\circ}$ ). The major reason was discussed earlier, i.e. the use of steady-state equations rather than time-dependent ones, thereby ignoring particle acceleration mechanisms, plasma oscillations and wave-particle interactions especially in the negative potential wells behind the satellite close to the wake axis (Samir and Wrenn, 1972; Gurevich and Pitaevskii, 1975; Al'pert, 1976). Another important reason for the discrepancy between theory and experiment is that in Parker's theory one ion equation is solved for a hypothetical ion with a mass equal to the mean ion mass of the ambient plasma which is reflected only in the values of the Mach number. This implies a constant mean ion mass. Realistically, separate equations would have to be used for the various constituents. In that case Gurevich et al. (1969) have shown that in the satellite wake close to the satellite surface the $\mathrm{H}^{+}$concentration substantially exceeds the $\mathrm{O}^{+}$concentration even if the ambient concentration ratio $\left|\mathrm{H}^{+}\right| /\left|\mathrm{O}^{+}\right|$is less than $1-10 \%$. Hence, the mean ion mass is in fact a function of position. These considerations play of course a more important role in the Explorer 31 data than they do in the AE-C data because of the higher altitude of the former (see Table 1) and the consequent higher relative $\mathrm{H}^{+}$density in the ambient plasma. Since Parker's theory underestimates the ion density in the wake near the body, it will therefore also underestimate the electron density which is confirmed by Fig. 8. An additional reason why the calculated electron depletion is so much larger than the measured one, especially for large angles of attack, is a geometrical one (see Fig. 3b) since the grid points of the calculation are much more effectively shielded from the plasma flow than the measurement points. This affects the ions directly and therefore indirectly the electrons. In summary, the comparison between the computed values of the density ratio $\left|\left(N_{+}(\theta)\right) /\left(N_{+0}\right)\right|$ and $\left|\left(N_{e}(\theta)\right) /\left(N_{e 0}\right)\right|$ and measured $\left|\left(I_{+}(\theta)\right) /\left(I_{+0}\right)\right|$ and $\left|\left(N_{e}(\theta)\right) /\left(N_{e 0}\right)\right|$ shows the quantitative degree of agreement obtained. It appears that despite semitechnical limitations imposed on such a comparison the major causes for the discrepancies between theory and experiment in the maximum wake zone seem to be due to the use of steadystate equations and a single ion equation. The present study shows that in the angle of attack range $90<\theta<145^{\circ}$ the agreement between theory and the AE-C ion measurements is fairly good and that even the agreement between the neutral approximation (i.e. 0th iteration) and the measurements is not too bad (see Figs. 6 and 7). The great computational effort of 15 iterations thus yields only a relatively small improvement. In the deep wake region $\left(\theta>145^{\circ}\right)$ theory and experiment differ by several orders of magnitude, and it is therefore unlikely that more sophisticated mathematical procedures applied to a steady-state theory with a single ion equation (for a hypothetical ion of mass equal to the mean ion mass in the undisturbed plasma) will yield significantly better agreement between theory and cxperiment. Wc submit that time-dependent solutions for the Vlasov-Poisson equations should be sought. In this way the effects of some relevant and important physical processes (e.g. particle acceleration, plasma oscillations, wave-particle interactions) on the wake structure will be included. Furthermore, even in cases where a steady-state solution may be an applicable approximation (Gurevich and Pitaevski, 1975; Al'pert, 1976) the various ion constituents should be treated separately rather than as a single ion with a fixed mean ion mass.

Acknowledgement-The cooperation of Dr. Lee Parker is gratefully acknowledged in putting his computer program at our disposal. We also acknowledge valuable discussions with Dr. N. H. Stone of the Space Sciences Laboratory, Marshall Space Flight Center. The help of D. Widjaja in carrying out the computer runs and plotting large numbers of profiles is gratefully acknowledged. This research has been supported by the National Aeronautics and Space Administration under Grant NGR 23-005-320 and the National Science Foundation under Grant ATM 76-19790. The computations were carried out at the Computing Facility of the National Center for Atmospheric Research, Boulder, Colorado.

\section{REFERENCES}

Al'pert, Ya. L. (1976). Wave-like phenomena in the near earth plasma and interactions with man-made bodies. Handbuch der Physik (Ed. by S. Flugge) Geophysics III. Part V, p. 217.

Brace, L. H., Theis, R. F. and Dalgarno, A. (1973). The cylindrical electrostatic probes for Atmosphere Explorer-C, -D and -E. Radio Sci. 8(4), 341.

Brinton, H. C., Scott, L. R., Pharo, M. W. and Coulson, J. T. C. (1973). The Bennet ion-mass spectrometer on Atmosphere Explorer-C and -E, Radio Sci. 8(4), 323.

Call, S. M. (1969). The interaction of a satellite with the ionosphere, Report No. 46, Plasma Laboratory, Columbia Univ.

Fournier, G. (1971). Collisionless plasma flow around a cylinder considering applications to ionospheric probes (in French). Publ. No. 137, O.N.E.R.A. 
Grabowsky, R. and Fischer, T. (1975). Theoretical density distribution of plasma streaming around a cylinder. Planet. Space Sci. 23, 287.

Gurevich, A. V., Pitaevskii, L. P. and Smirnova, V. V. (1969). Ionospheric aerodynamics. Space Sci. Rev. 9, 805 .

Gurevich, A. V., Pariskaya, R. V. and Pilaevskii, I.. P. (1973). Ion acceleration upon expansion of a rarefied plasma. Soviet Phys. JETP 36(2), 274.

Gurevich, A. V. and Dimant, Ya. S. (1975). Flow of a rarefield plasma around a disc. Geomagnetism and Aeronomy 15(2), 183.

Gurevich, A. V. and Pitaevski, L. P. (1975). Non-linear dynamics of a rarefied ionized gas. Prog. Aerospace Sci. 16, 227.

Liu, V. C. (1969). Ionospheric gas dynamics of satellites and diagnostic probes. Space Sci. Rev. 9, 423.

Liu, V. C. (1975). On ionospheric aerodynamics. Prog. Aerospace Sci. 16(3), 273.

Parker, L. W. (Feb. 1976). Computation of collisionless steady-state plasma flow past a charged disc. Report, NASA CR-144159.

Parker, L. W. (Feb. 1977). Calculation of sheath and wake structure about a pillbox-shaped spacecraft in a flowing plasma. Proc. Spacecraft Charging Technology Conf. (Ed. by C. P. Pike and R. R. Lovell) Joint Air Force-NASA Reporl AFGI-TR-77-0051 and NASA TMX-73537, p. 331.

Samir, U. and Jew, H. (1972). Comparison of theory with experiment for electron density distribution in the near wake of an ionospheric satellite. J. geophys. Res. 77 , 6819.

Samir, U. and Wrenn, G. L. (1972), Experimental evidence of an electron temperature enhancement in the wake of an ionospheric satellite. Planet. Space Sci. 20, 899.

Samir, U., First, M., Maier, E. J. and Troy, B. E. (1975). A comparison of the Gurevich et al. and Liu-Jew models for the ion flux around a satellite. J. atmos. terr. Phys. 37, 577.
Samir, U., Gordon, R., Brace, L. and Theis, R. (1979). The near wake structure of the Atmosphere Explorer $C$ (AE-C) Satellite: A parametric investigation. J. geophys. Res. 84(2), 513.

Samir, U., Kaufman, Y. J., Brace, L. H. and Brinton, H. C. (1980). The dependence of ion density in the wake of the AE-C satellite on the ratio of body size to Debye length in an $\left(\mathrm{O}^{+}\right)$dominated plasma. J. geophys. Res. 85, 1769.

Willmore, A. P. (1970). Electron and ion temperatures in the ionosphere. Space Sci. Rev. 11, 607.

Wrenn, G. L. (1969). The Langmuir plate and spherical ion probe experiments aboard the Explorer 31, Proc. IEEE 57, 1072.

\section{APPENDIX}

A question of "technical" nature in comparing measurements with theoretical models which employ iteration techniques and grid meshes for the mathematical solutions is that of fixing the boundary at "infinity". Namely, it is of practical interest to investigate whether the location of the boundary at "infinity" in the calculations has indeed a significant influence on the comparison with experiment. Figure A-1 compares the angle of attack profiles of AE-C case 4 for $Z_{\max }=6$ and 12 . All distances are normalized with respect to the satellite radius $R_{0}$. There is a $12 \%$ difference between the points at $130^{\circ}$, and otherwise the two profiles are in complete agreement even though the distance from the body to the boundary has been doubled. Hence, if $Z_{\max }=6$, the results relatively close to the body are fairly insensitive to changes in $Z_{\max }$. Similar results are expected for the other cases. In all of our calculations $Z_{\max }$ has been chosen to be 6 .

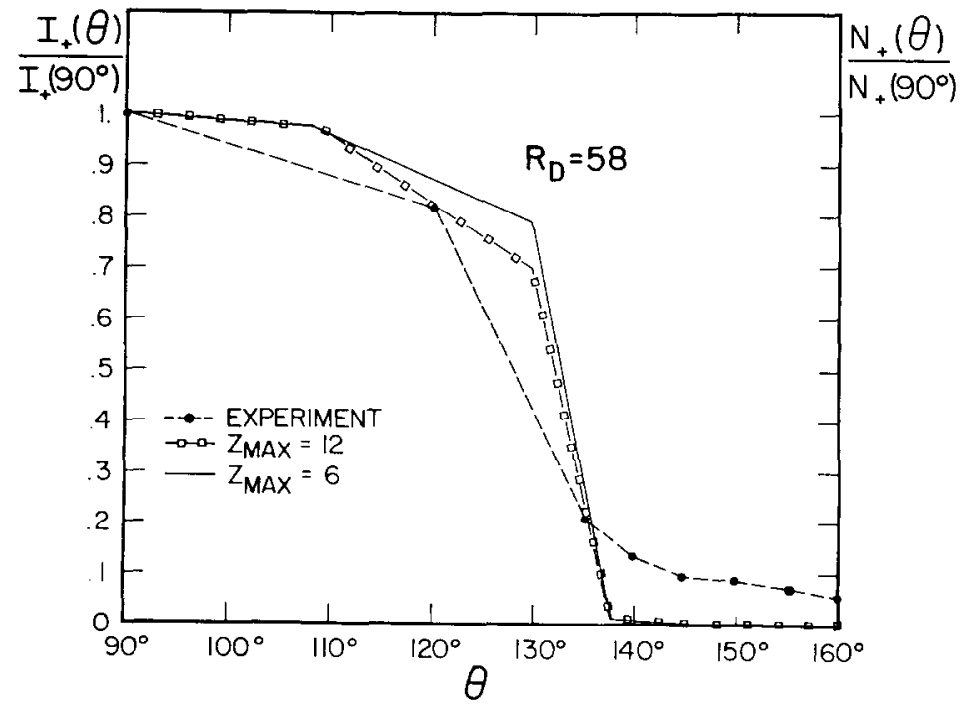

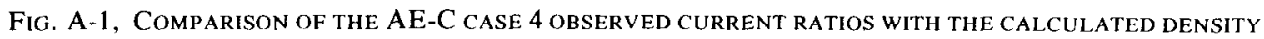
RATIOS OBTAINED WITH $Z_{\max }=6$ AND $Z_{\max }=12$. 